\title{
Les mésaventures éditoriales de Toine ou comment déculturer un récit de Maupassant.
}

Un dossier iconographique

\section{Jean-Marie Privat et Marie-Christine Vinson}

\section{(2) OpenEdition}

\section{Journals}

Édition électronique

URL : http://journals.openedition.org/pratiques/1896

DOI : 10.4000/pratiques.1896

ISSN : 2425-2042

Éditeur

Centre de recherche sur les médiations (CREM)

Édition imprimée

Date de publication : 15 décembre 2011

Pagination : 273-293

\section{Référence électronique}

Jean-Marie Privat et Marie-Christine Vinson, « Les mésaventures éditoriales de Toine ou comment déculturer un récit de Maupassant. », Pratiques [En ligne], 151-152 | 2011, mis en ligne le 13 juin 2014, consulté le 14 novembre 2019. URL : http://journals.openedition.org/pratiques/1896 ; DOI : 10.4000/ pratiques. 1896 


\title{
Les mésaventures éditoriales de Toine ou comment déculturer un récit de Maupassant. Un dossier iconographique
}

\author{
Jean-Marie Privat \\ Université Paul Verlaine - Metz, CELTED, EA 3474 \\ Marie-Christine Vinson
}

Université Henri Poincaré Nancy (IUFM de Lorraine), CELTED, EA 3474

Nous avons choisi un récit de Maupassant - Toine, 1885 - pour analyser à partir d'un corpus d'illustrations échelonnées sur presque un siècle comment l'iconographie traduit ou programme des lectures qui peuvent tendre rapidement à la fois à académiser et à déculturer un texte, depuis sa première publication en livre (1886) jusqu'aux rééditions des années 1970 . Voici le début de l'histoire ${ }^{(1)}$ :

«On le connaissait à dix lieues aux environs le père Toine, le gros Toine, Toinema-Fine, Antoine Mâcheblé, dit Brûlot, le cabaretier de Tournevent [...]. Sa Fine, c'était son cognac, bien entendu.

Depuis vingt ans il abreuvait le pays de sa Fine et de ses Brûlots, car chaque fois qu'on lui demandait. - Qu'est-ce que j'allons bé, pé Toine ? Il répondait invariablement : - Un brûlot, mon gendre, ça chauffe la tripe et ça nettoie la tête ; y a rien de meilleur pour le corps.

Il avait aussi cette coutume d'appeler tout le monde "mon gendre", bien qu'il n'eût jamais eu de fille mariée ou à marier.

(1) Maupassant, Toine, Edition établie, présentée et annotée par Louis Forestier, Paris, Gallimard / Folio, 1991, pp. 29-41. Ce récit a paru d'abord dans le Gil Blas du 6 janvier 1885, sans illustration. Toine est par ailleurs le titre éponyme d'un recueil de dix-huit nouvelles que Maupassant a rassemblées et publiées sous forme de livre dès 1886 . Une deuxième édition légèrement remaniée et augmentée a été publiée dès fin 1886, toujours à Paris chez Marpon et Flammarion, dans leur Bibliothèque illustrée ( 5 francs le volume). C'est dire que Toine, le texte et/ou le recueil, eurent d'entrée un franc succès : «Je viens de recevoir avis de Marpon que Toine et les Contes du jour et de la nuit, sont l'un au dixième mille, l'autre au onzième [...] », Correspondance, Lettre du 20 octobre 1887). Pour tout complément d'information sur Toine, de 1'édition originale jusqu'aux diverses adaptations contemporaines, se reporter aux sites spécialisés / www.maupassantiana.fr/ et / maupassant.free.fr/ 
Ah! oui, on le connaissait Toine Brûlot, le plus gros homme du canton, et même de l'arrondissement. Sa petite maison semblait dérisoirement trop étroite et trop basse pour le contenir [...]. Il y entrait chaque fois que se présentait un consommateur, car Toine-ma-Fine était invité de droit à prélever son petit verre sur tout ce qu'on buvait chez lui.

Son café avait pour enseigne : "Au Rendez-vous des Amis”, et il était bien, le pé Toine, l'ami de toute la contrée [...]. Il mangeait et il buvait comme dix hommes ordinaires $[\ldots] .{ }^{(2)}$

Point n'est besoin d'être expert en ethnocritique pour voir se dessiner d'entrée un univers de sociabilité rurale et populaire, festive et masculine, une culture comique voire farcesque du «bas » matériel et corporel ${ }^{(3)}$, un petit monde de carnaval où le héros est un géant gargantuesque et les engendrements aussi ventripotents qu'imaginaires et parodiques.

C'est en tout cas dans cet esprit que Mesplès ${ }^{(4)}$ illustre en 1886 l'édition originale de Toine (ill. 1). Le corps du cabaretier est un ventre énorme et proéminent posé sur une chaise comme un vivant et hilare mannequin de carnaval. Et ses compères sont croqués à leur tour en farceurs rigolards et aux trognes animales :

«Ils étaient trois qui reparaissaient tous les jours : Célestin Maloisel, un grand maigre, un peu tordu comme un tronc de pommier, Prosper Horslaville, un petit sec avec un nez de furet, malicieux, futé comme un renard, et Césaire Paumelle, qui ne parlait jamais, mais qui s'amusait tout de même. »

Quant à sa femme, la mé Toine, « la vieille», âpre au gain et rude au travail, figure de Carême et prophète de malheur, elle est en tout point le double inversé du pé Toine, ce roi fainéant d'un pays de Cocagne :

"C'était une grande paysanne, marchant à longs pas d'échassier, et portant sur un corps maigre et plat une tête de chat-huant en colère. Elle passait son temps à élever des poules dans une petite cour [...]. Fâchée contre le monde entier, elle en voulait principalement à son mari. Elle lui en voulait de sa gaieté, de sa renommée, de sa santé et de son embonpoint. Elle le traitait de propre à rien, parce qu'il gagnait de l'argent sans rien faire, de sapas, parce qu'il mangeait et buvait comme dix hommes ordinaires, et il ne se passait point de jour sans qu'elle déclarât d'un air exaspéré :

- Ça serait-il point mieux dans l'étable à cochons nu quétou comme ça ? C'est que d'la graisse que ça en fait mal au cœur.

Et elle lui criait dans la figure :- Espère, espère un brin ; j'verrons c'qu'arrivera, j'verrons ben ! Ça crèvera comme un sac à grain, ce gros bouffi ! [...] Et les con-

(2) Les éditions du court texte isolé de Toine ou du recueil du même nom étant aussi nombreuses qu'aisément accessibles (y compris sur Internet), nous ne référerons pas systématiquement nos citations du récit.

(3) M. Bakhtine, "Le "bas" matériel et corporel chez Rabelais ", L'œuvre de François Rabelais et la culture populaire au Moyen Age et sous la Renaissance, Paris, Gallimard, 1970, pp. 366-432.

(4) Paul-Eugéne Mesplès (1849-1924). Peintre, lithographe et graveur français. Fréquente l'atelier de Gérôme. Il illustre des romans dont La Pipe cassée de Vadé et le théâtre de Beaumarchais. Créateur de bijoux à ses débuts, puis caricaturiste, il fut également dessinateur au Museum d'histoire naturelle. Expose au Salon de 1880. Collabore au Chat Noir, L'Illustration, Le Monde illustré. 


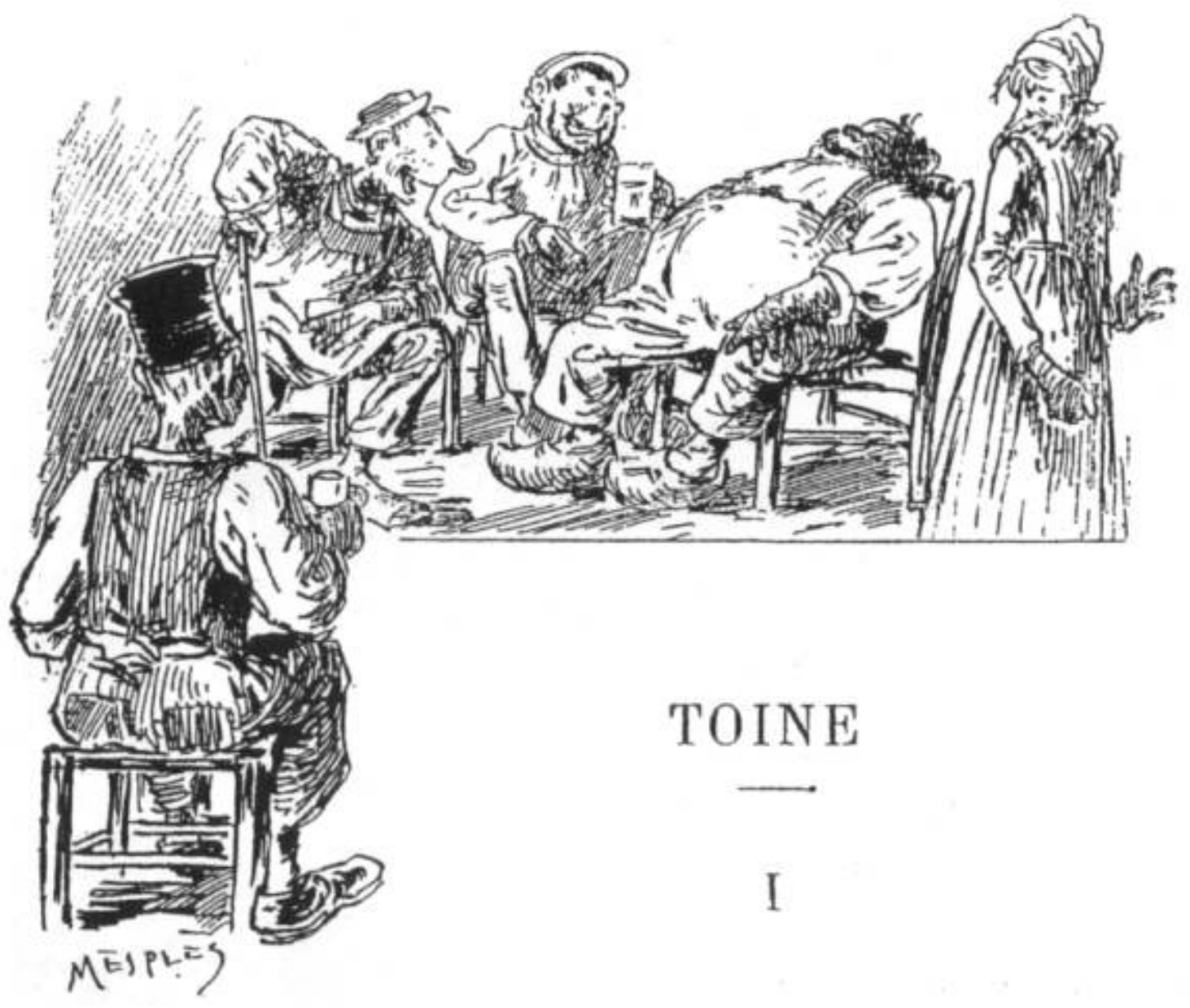

Illustration 1 : 1886. Toine, Edition originale, Illustration P.-E. Mesplès, Paris, Bibliothèque illustrée à 5 francs le volume, Marpon \& Flammarion, 1886. 
sommateurs tapaient du poing sur les tables en se tordant de joie, tapaient du pied sur la terre du sol, et crachaient par terre dans un délire de gaieté. La vieille furieuse reprenait : - Espère un brin... espère un brin... j'verrons c'qu'arrivera... ça crèvera comme un sac à grain... Et elle s'en allait furieuse, sous les rires des buveurs. »

Cette galerie de grotesques en sabots dessinés d'un trait à l'expressivité bouffonne sinon outrancière illustre nous semble-t-il assez précisément les données textuelles et l'atmosphère de carnavalisation naturaliste typique de l'écriture de Maupassant ${ }^{(5)}$.

Dès 1903, avec les illustrations de V. Rottembourg ${ }^{(6)}$, on change visiblement de registre plastique et d'univers social (ill. 2). La parade farcesque et expressionniste des bougres de hameaux se mue en une visite des amis au bon Toine alité et serein, souriant et paisible, à peine goguenard. La mère Toine est habillée comme une paysanne endimanchée, digne et offusquée, en lutte sourde, douloureuse et muette, avec le petit club des hommes. Le dessin est cette fois d'une sobriété petite bourgeoise pourrait-on dire : le trait contenu, mesuré, assagi se conjugue au réalisme discret des détails pour peindre une scène d'intérieur où s'affrontent sur un mode allusivement dramatique des regards amusés ou peut-être sournois et des figures inertes, pensives ou douloureuses. Cette vision moralisante et psychologisante des rapports sociaux sur un fond de tristesse marquée et de sourires entendus ne correspond ni à la bavarde agressivité théâtrale ni à la dynamique de la transposition littéraire (et presque littérale) du combat rituel de Pé-Carnaval et de Mé-Carême :

« On apportait une planche de la cour, on la posait au bord du lit et on jouait aux dominos pardi, et on faisait de rudes parties, depuis deux heures jusqu'à six.

Mais la mère Toine devint bientôt insupportable. Elle ne pouvait tolérer que son gros faigniant d'homme continuât à se distraire, en jouant aux dominos dans son lit ; et chaque fois qu'elle voyait une partie commencée, elle s'élançait avec fureur, culbutait la planche, saisissait le jeu, le rapportait dans le café et déclarait que c'était assez de nourrir ce gros suiffeux à ne rien faire sans le voir encore se divertir comme pour narguer le pauvre monde qui travaillait toute la journée. »

Les illustrations (en couleurs) d'A. Robaudi ${ }^{(7)}$ (1923) accentue le pittoresque

(5) Jean-Marie Privat, « Toine, ou le géant exhibé », Bulletin Flaubert-Maupassant, 13, 2003, pp. 15-26.

(6) Valérie Rottembourg, dessinatrice française née à Paris. Elle a illustré de 1901 à 1913 des classiques de la littérature française pour des éditeurs parisiens (Balzac, Marivaux, Maupassant...) Ce sont les seules informations que donne Le Dictionnaire des illustrateurs (1890-1945) de Marcus Osterwalder publié par Ides et Calendes, Neuchâte1, 1992, p. 998.

(7) Alcide Théophile Robaudi, peintre et illustrateur, né à Nice en 1850, mort à Paris en 1928. Peintre de genre plus académique que son maître Gérôme, il est aussi influencé par le courant orientaliste. Il débute au Salon de 1874 où il présente une scène de genre. Portraitiste, paysagiste, il reçoit la mention «honorable » en 1884. Par la suite il sera surtout reconnu comme l'illustrateur des éditions de luxe (Conquet, Hachette, Calmann-Lévy, Lemerre, Ferroud) d'ouvrages d'écrivains célèbres (Balzac, Musset, A. Dumas, G. Sand, P. Verlaine). En 1921, il signera une couverture de programme polychrome sur le thème du Carnaval de Nice pour le Casino municipal de Nice. Il est l'auteur d'affiches pour le P.L.M. ou pour l'emprunt national de 1916. Il réalise des séries de dessins sur le thème d'“idylles champêtres" et des portraits de jeunes filles normandes dans les vergers. 


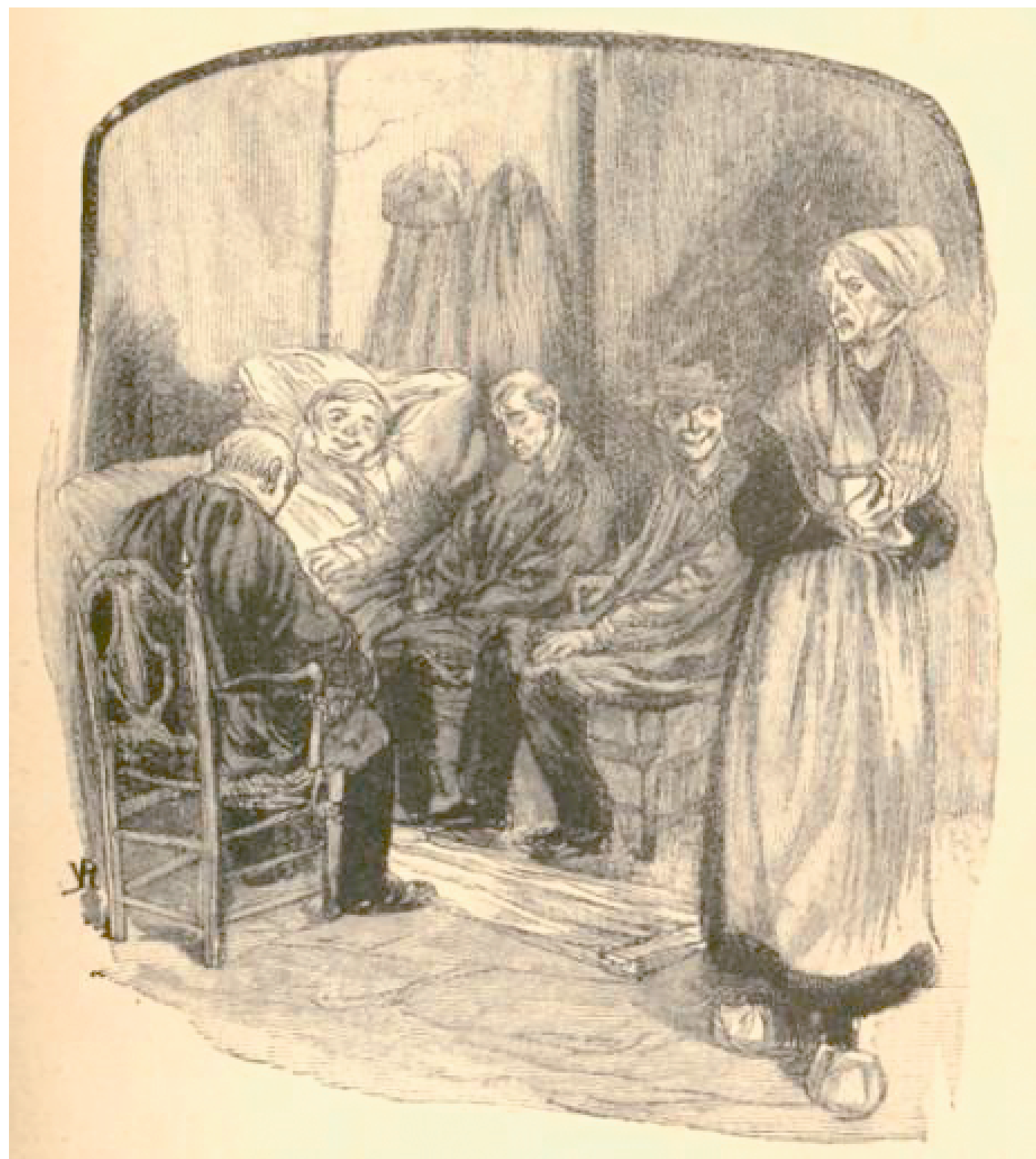

Illustration 2 :1903. Toine, Illustration V. Rottembourg, gravée sur bois par G. Lemoine, Paris, Ollendorff, Euvres complètes illustrées de Guy de Maupassant, 1903. 
rustique, souriant et inoffensif de Toine (ill. 3). A l'enseigne du « Rendez-vous des Amis » il ne reste que le portrait d'un couple traditionnel, l'homme de bonne stature, aussi accueillant qu'oisif sur le pas d'une porte grande ouverte, une vigne vierge maigrichonne taillée à la diable et quelques rares poules ou poussins qui picorent en liberté, une femme à l'arrière-plan, sévère et laborieuse. Une vue pittoresque sur une campagne aux mœurs stéréotypées, où la bonhomie tient lieu de véritable richesse et où l'éternel partage du travail tient lieu d'ordre du monde. La culture folklorique et festive du récit de Maupassant a disparu, le jeu avec la familiarité populaire de la boutique s'est affadi, toute forme d'excentricité visuelle ou comportementale a disparu, la transgression des barrières anthropologiques aussi bien entendu (plus de mascarades animales). Seul demeure l'ancrage dans un univers rural, plus conventionnel qu' ethnotypique et un personnage somme toute très convenable et non plus débordant de jovialité avinée et marchande :

« Il avait une manière de blaguer les gens sans les fâcher, de cligner de l'œil pour exprimer ce qu'il ne disait pas, de se taper sur la cuisse dans ses accès de gaieté [...]. Il buvait tant qu'on lui en offrait, et de tout, avec une joie dans son œil malin, une joie qui venait de son double plaisir de se régaler d'abord et d'amasser des gros sous, ensuite, pour sa régalade. »

Cette civilisation iconique des mœurs contrevient aux évaluations empathiques du narrateur - «C'était une curiosité rien que de le regarder boire » ou « Il aurait fait rire le diable, ce malin-là »- et aux conduites décivilisées d'Antoine Mâcheblé (sa goinfrerie, son ivrognerie, son animalité), " ce gros faigniant, qu'i faut nourrir, qu'i faut laver, qu'i faut nettoyer comme un porc. » Sont pareillement censurées les manifestations du corps de commediante, corps excessif et expressif - « Il avait une manière de cligner de l'œil pour exprimer ce qu'il ne disait pas [...]. Il vous tirait le rire du ventre malgré vous »-et du corps collectif des Sancho Pancha de hameau normands - « Les trois amis de Toine riaient à suffoquer, toussant, éternuant, poussant des cris [...]». Cette acculturation moralisante ou bien pensante du récit de Maupassant passe aussi sous silence, évidemment, la violence physique - « La mère Toine [...] frémissant de fureur [...], se mit à lui taper de grands coups sur le ventre, comme lorsqu'elle lavait le linge au bord de la mare. Ses mains tombaient l'une après l'autre avec un bruit sourd, rapides comme les pattes d'un lapin qui bat du tambour. " ${ }^{(8)}$ - et reste allusive sur la violence symbolique - « Toine fut vaincu [...]. La vieille le privait de nourriture avec férocité [...]. » La dimension discrètement politique de la nouvelle est elle aussi absente - «Quand on donnait un repas à Fécamp chez les gens de la haute, il fallait, pour que le dîner fût goûté, qu'on y mangeât une pensionnaire (une poule) de la mè Toine. » Sans doute dans l'après-guerre le marché éditorial et le légitimisme cultivé du lectorat sont-ils spontanément plus favorable à l'imagerie ruraliste de l'époque et à l'idéologie des relations harmonieuses entre

(8) Ce passage évoque La farce du cuvier, pièce du théâtre comique, populaire et profane des $\mathrm{XV}^{\mathrm{e}}-\mathrm{XVI} \mathrm{I}^{\mathrm{e}}$ siècles, un autre monde (provisoirement) à l'envers où violence physique, violence verbale, violence conjugale et violence symbolique sont au principe même de l'action dramatique. 


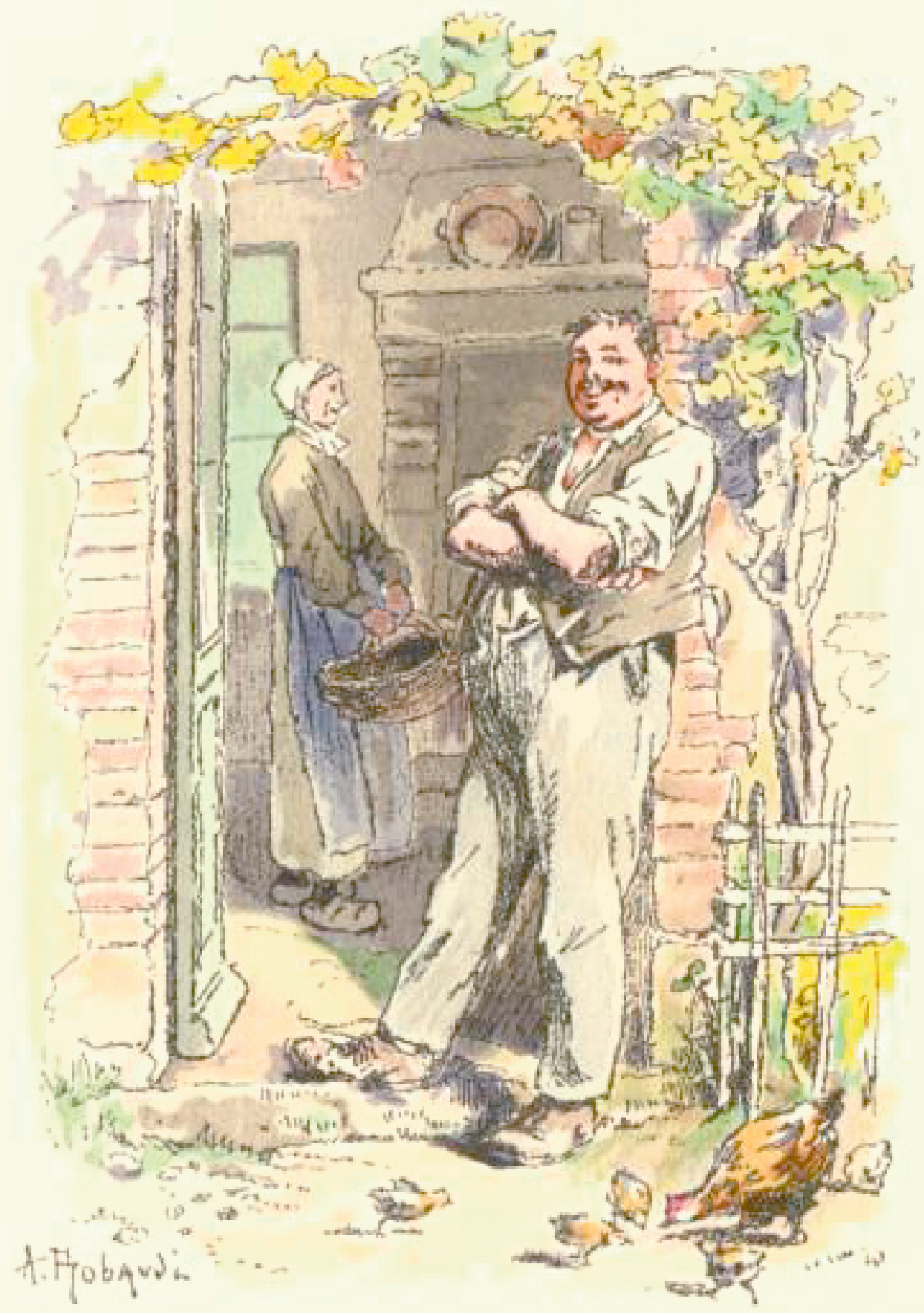

Illustration 3 :1923. Toine suivi de Histoire d'une fille de ferme, Illustrations en couleurs par A. Robaudi, Paris, A. Ferroud \& F. Ferroud, 1923 
ville et campagne, paysannerie et bourgeoisie provinciale (bien) française qu'aux belligérances socio-symboliques.

Quand P. Falké ${ }^{(9)}$ illustre en 1938 les Euvres complètes de Guy de Maupassant pour La Librairie de France (ill. 3), le dessin gagne en modernité stylistique mais le regard sur le hameau de Tournevent et ses drôles d'habitants reste pittoresque comme si les cultures subalternes proches - aussi drôles que puériles, fantaisistes que régressives - étaient d'inépuisables réserves d'altérité culturelle où le lecteur serait pris entre une certaine et ludique fascination et une réelle distance amusée :

« La classe paysanne, sans doute parce qu'elle ne s'est jamais donné ou qu'on ne lui a jamais donné le contre-discours capable de la constituer en sujet de sa propre vérité, est l'exemple par excellence de la classe objet [...]. Simple prétexte à préjugés favorables ou défavorables [...], la forme fondamentale du profit de distinction assigne aux paysans leurs réserves, où ils auront tout le loisir de danser et de chanter leurs bourrées et leurs gavottes [...]. Ce n'est pas d'aujourd'hui que les paysans, sans cesse affrontés à la domination inséparablement économique et symbolique de la bourgeoisie urbaine n'ont pas d'autre choix que de jouer [...] l'une ou l'autre des figures du paysan, celle du paysan respectueux [...] parlant de sa terre, de sa maison et de ses bêtes avec des accents de rédaction d'école primaire, ou celle du paysan [...] qui sait prendre son temps et cultiver le silence et qui étonne [...] par sa profonde sagesse, venue on ne sait d'où, ou encore celle du paysan empaysanné qui assume [...] le rôle du « simple », du « cul-terreux », du bon sauvage ou même celle du braconnier, parfois un peu sorcier, qui épate autant [...] par son habileté à découvrir les champignons ou à tendre des lacets que par ses talents de rebouteux ou ses croyances d'un autre âge. " ${ }^{(10)}$

P. Falké propose un monde hybride en fait qui mêle le dessin à la diable d'une grosse tête rieuse de carnaval - « une joie dans son œil malin »... - et le trait du dessinateur de Montmartre pour les compères du père Toine. Un peu comme si l'artiste réservait sa verve graphique populaire et gentiment caricaturale au saltimbanque de Tournevent et son crayon moderniste et presque cubiste dans le traitement de ses farceurs d'acolytes (ill. 4). Ce collage ou ce dédoublement stylistique vise sans doute à placer dans une proximité complice les lecteurs urbains du récit, Antoine Mâcheblé continuant de son côté d'offrir le spectacle d' « un drôle d'homme »d'un lointain hameau normand.

(9) Jean (Pierre) Falké (1884-1947), dessinateur de presse, illustrateur (L'Almanach des saisons) et humoriste (militaire). Il a notamment travaillé pour les journaux Le Rire et Le Crapouillot. Il deviendra un des membres estimé du Salon de l'Araignée. Grand voyageur, il se spécialisera dans les vues maritimes. P. Falké a illustré plus de soixante livres chez de grands éditeurs parisiens (Fayard, Librairie de France, etc.).

(10) P. Bourdieu, "Une classe objet», Actes de la recherche en sciences sociales, vol. 17-18, 1977, Paris, Seuil, pp. 2-5. Voir aussi pour une inscription de notre sujet dans une histoire sociale, culturelle, artistique et idéologique longue, R. R. Brettell et C. Brettell, Les peintres et le paysan au XIX siècle, Genève, Skira, 1983 ; H. Bernard, La terre toujours réinventée. La France rurale et les peintres 1920-1955. Une histoire de l'imaginaire, Lyon, P.U.L., 1990 et E. Weber, La fin des terroirs. La modernisation de la France rurale 18701914, Paris, Fayard / Editions Recherches, 1983. 


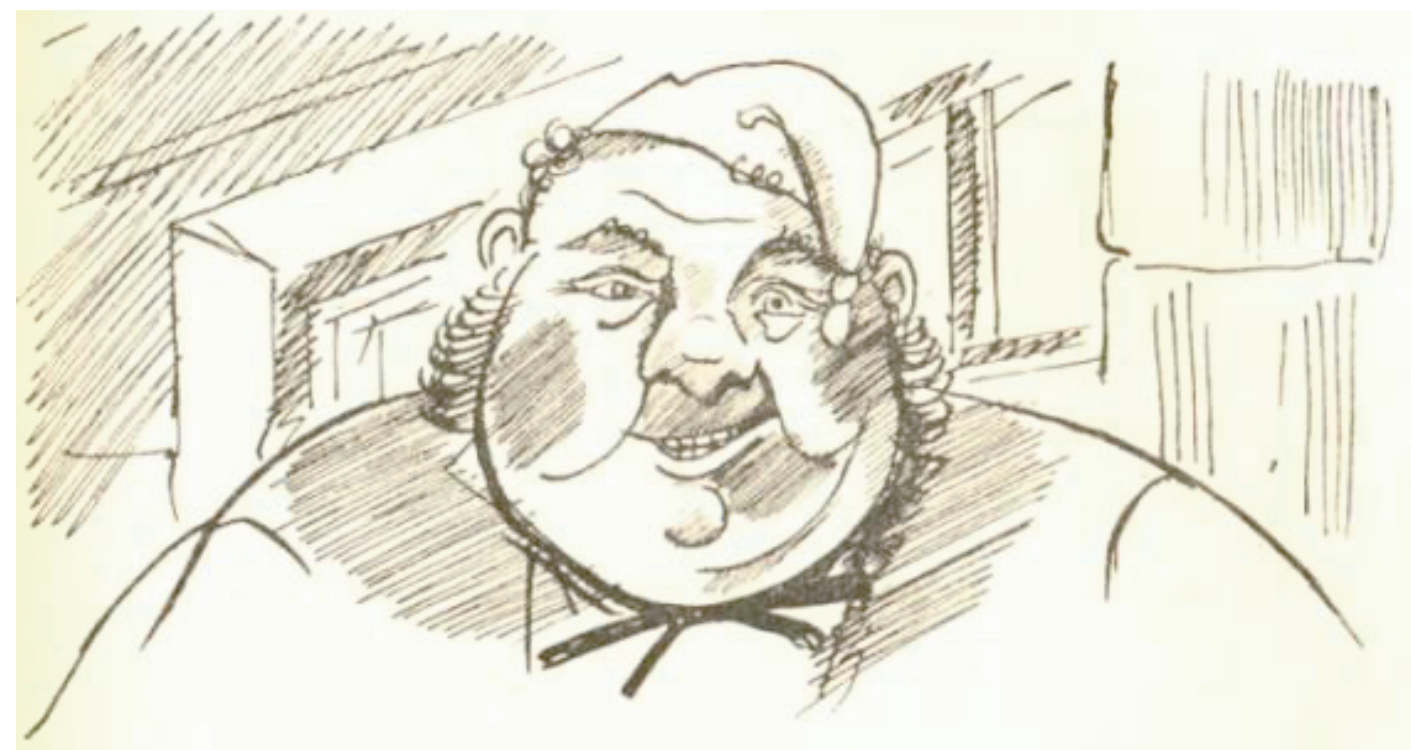

Illustration 4 : 1938. Toine, Illustrations P. Falké, Paris, Librairie de France, Euvres complètes illustrées de Guy de Maupassant, V, 1938. 
La dernière image de notre corpus ${ }^{(11)}$ est une illustration de Jean-François Debord $^{(12)}$ pour l'édition de Toine (1970) chez H. Piazza, Paris. Notre récit est entré dans le monde de la culture artistique moyenne (ill. 5). Toine fait en effet l'objet cette fois d'un lavis publié à l'enseigne de L'Edition d'art. Il s'agit de la transposition très esthétisante du récit dans l'univers pictural et personnel de l'artiste. La scène de genre s'est largement dépaysannisée (le sol terreux est lui-même désormais un pavé à la géométrie alternée des plus sobrement classiques), l'enluminure rustique et festive « de rouge et de bleu » s'est diluée dans une palette aux teintes estompées, les personnages ont perdu toute épaisseur charnelle, et le drame domestique muet - sous forme d'une tension psychologique et relationnelle - s'esquisse sis entre un pot de fleurs (posé sur un rebord de fenêtre dans le vague d'une couleur délavée) et un chat sage et rêveur, au pied d'un lit à baldaquin :

«-C'est ça qui me fait deuil, mon gendre, de n'pus goûter d'ma Fine, nom d'un nom. L'reste, j'men gargarise, mais de ne point bé mé ça fait deuil.

Et la tête de chat-huant de la mère Toine apparaissait dans la fenêtre. Elle criait : - Guêtez-le, guêtez-le, à c't'heure ce gros faigniant [...].

Et quand la vieille avait disparu, un coq aux plumes rouges sautait parfois sur la fenêtre, regardait d'un œil rond et curieux dans la chambre, puis poussait son cri sonore. Et parfois aussi, une ou deux poules volaient jusqu'au pied du lit, cherchant des miettes sur le sol. »

Un « pauvre hameau paysan », un « colosse » couché sur sa «paillasse» dans une petite chambre bientôt envahie par les «buveurs »? Le monde de Toine s'est à la fois rapetissé (les interactions personnelles se sont substituées à la culture locale et communautaire) et moralisé, entre stéréotype littéraire psychologisant et ethnotype culturel dévitalisé.

Les lectures illustrées de Maupassant ont ainsi très vite perdu leurs puissances empathiques et festives pour devenir tristement muséales (où sont passés «ses accès de gaieté qui vous tirait le rire du ventre malgré vous, à tous les coups »?). Comme s'il fallait tenir à distance prudente la polyphonie du récit et le dialogisme qu'il crée et recrée sans cesse entre l'univers de Toine et l'univers du lecteur, dès l'incipit - «On le connaissait ». Cette participation ludiquement offerte

(11) On retrouvera aisément dans les nombreuses rééditions disponibles de Toine les références aux adaptations cinématographiques ou aux téléfilms dont ce récit a fait l'objet. Parmi les éditions scolaires illustrées, citons Guy de Maupassant, «Toine », Histoires douces amères, Illustrations de Ph. Dumas, Paris, L'Ecole des loisirs / Classiques, 2008 (1981), pp. 4459 et Maupassant, Toine et autres contes normands. Flammarion, Paris Du texte à l'écran, Paris, Flammarion / Etonnants classiques, 2007 (dossier iconographique). On retrouvera des illustrations (colorisées parfois) de V. Rottembourg et de P. Falké dans Nouvelles de Maupassant, pp. 16-19 à l'adresse medias.hachette-education.com/media/.../029/482955873.PDF

(12) Jean-François Debord (1938-...), peintre et ancien professeur de morphologie à l'Ecole nationale des beaux-arts de Paris et à l'Ecole de kinésithérapie de Paris. Ancien assistant de l'affichiste Cassandre, il est l'initiateur de la première exposition des ovales de Duchenne de Boulogne au musée Nicéphore Niépce en 1984. Il a inspiré à Agnès Maupré l'album de bande dessinée Petit Traité de morphologie d'après les cours donnés par Jean-François Debord à l'école des Beaux-Arts de Paris de 1978 à 2003 (éd. Futuropolis, 2008). Une de ses devises, empruntée à Cassandre, est : «Lorsqu'on dessine une courbe, il faut penser la droite qui la sous-tend. » 


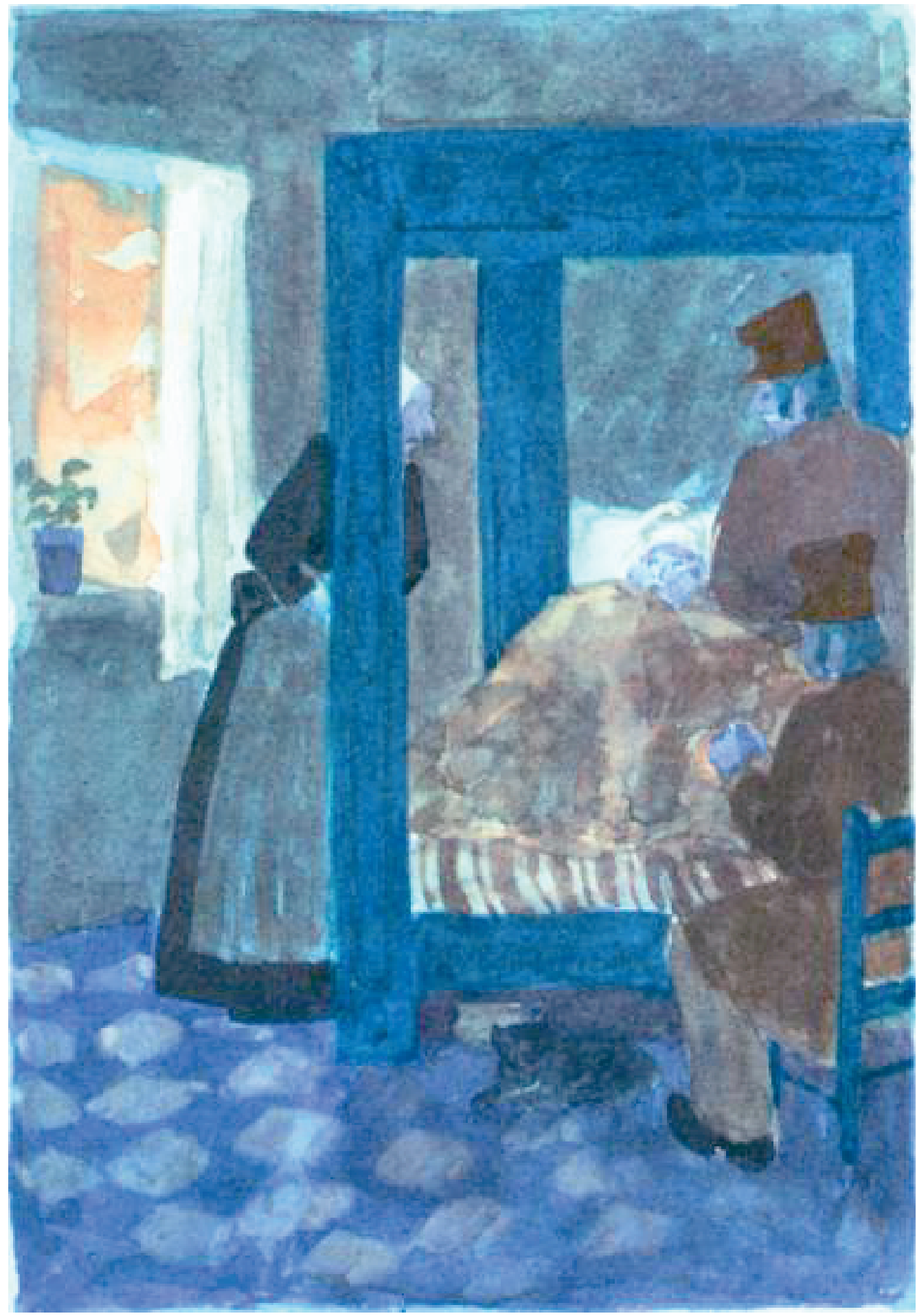

Illustration 5 :1970. Toine, Illustrations de Jean-François Debord, Paris, L'Edition d'Art H. Piazza, 1970. 
est résumée en son parler paysan par la proposition d'un des compères de Toine (à l'adresse de sa femme...) : «si j'étais de vous ».

Comme s'il fallait toujours choisir l'isotopie réaliste et monologique contre les possibles cognitifs de l'univers des contes et contre les histoires impossibles propres au grotesque carnavalesque, la certitude du mimétisme référentiel contre l'hésitation du narrateur et la fiction d'un imaginaire textuel ensauvagé et anamorphique :

— «Elles étaient là, ces maisons, blotties dans ce ravin couvert d'herbe et d'ajonc, derrière la courbe qui avait fait nommer ce lieu Tournevent. Elles semblaient avoir cherché un abri dans ce trou comme les oiseaux qui se cachent dans les sillons [...]. »

- Sa petite maison semblait dérisoirement trop étroite et trop basse pour le contenir, et quand on le voyait debout sur sa porte où il passait des journées entières, on se demandait comment il pourrait entrer dans sa demeure. Il y entrait chaque fois que se présentait un consommateur, car Toine-ma-Fine était invité de droit à prélever son petit verre sur tout ce qu'on buvait chez lui. » (Nous soulignons les modalisateurs des points de vue antagonistes, leur coalescence belligérante en fait qui construit littérairement le double jeu narratif du texte.) ${ }^{(13)}$

Ce récit se prête à l'illustration non seulement parce qu'il adopte peu ou prou le cahier des charges de la narration réaliste - « [...] Toine-ma-Fine passa ses jours et ses nuits dans son lit qu' on ne retapait qu'une fois par semaine, avec le secours de quatre voisins qui enlevaient le cabaretier par les quatre membres pendant qu' on retournait sa paillasse. »- mais encore parce que l'écrivain multiplie les images visuelles - «Sa maison semblait dérisoirement trop étroite et trop basse pour le contenir, et quand on le voyait debout sur sa porte [...]. »- et les références au théâtre ou à l'imagerie traditionnelle - «C'était une telle comédie qu'on aurait payé sa place de bon cœur »-«Il se mit à pousser des cris, et il lâcha le poussin qui courut sur sa poitrine. Le café était plein de monde. Les buveurs se précipitèrent, envahirent la chambre, firent cercle comme autour d'un saltimbanque, et la vieille [...] cueillit avec précaution la bestiole blottie sous la barbe de son mari » ou encore - «Il était devenu épais et gros [...], monstrueux et drôle » la mort prenant plaisir à «l'enluminer de rouge et de bleu ».

(13) Il n'est pas sûr que les perspectives de « l'archithématique » développées par F. Rastier à la fin d'une analyse subtilement scrutatrice de la texualité de Toine ne participent paradoxalement d'un effacement de la culture propre au récit, par une brusque montée en régime d'universalité cette fois : «L'homologation masculin :féminin :: visible : caché : froid : chaud est liée par exemple, comme l'a montré Granet, à la division du travail dans la Chine antique : les hommes, laboureurs ou bouviers, travaillant au-dehors, surtout pendant l'été ; les femmes, tisserandes ou fileuses, restant à l'intérieur. Au-delà, certaines homologations peuvent jouir d'une validité transculturelle, procédant de contraintes perceptives et cognitives. Les textes de la littérature dite universelle doivent sans doute une bonne part de leur universalité au caractère anthropologique des structures archithématiques qu'ils mettent en œuvre ", "Le Pé Poule », Sens et textualité, Paris, Hachette/U, 1989, p. 223 (réédition accessible et téléchargeable à l'adresse www.revue-texto.net/Parutions/Sens.../Rastier_sens_et_textualite.html). 
On se doute cependant que ce " réalisme abâtardi » et ce " grotesque mutilé ${ }^{(14)}$ ne sont pas propres aux illustrateurs modernes de Maupassant, même si la place dans le champ littéraire de l'auteur normand exposait ses contes ou nouvelles à une variante du droit de cuissage symbolique, une appropriation éthico-esthétisante, une douce violence idéologico-artistique. En fait, et plus généralement, la culture populaire comique apparaît aux approches historico-culturelles et historico-littéraires frappées de légitimisme académique comme « un ramassis de curiosités hétérogènes », un " assemblage de curiosités et d'obscénités » ${ }^{(15)}$. Aussi conçoit-on que « la puissante influence exercée par la culture comique populaire sur toutes les belles-lettres, sur la "pensée imagée" de l'humanité » soit encore largement sous-estimée, à tous les sens du terme ${ }^{(16)}$. Selon Bakhtine, cette tradition carnavalesque « avec sa gaieté dans les changements et sa joyeuse relativité rapprochant au maximum le monde de l'homme et l'homme de l'homme » (Toine tel un actant de carnaval sera tour à tour ou simultanément dans le récit un colosse et un enfant, un mari et une poule, un four et un lapin, etc.), s'oppose « au sérieux officiel, morose, monologique et dogmatique » des vérités officielles et dominantes ${ }^{(17)}$. Il semble bien en effet que surtout à partir de 1903 nos illustrateurs aient adopté un point de vue proche de ce "sérieux"... de Carême. Ce sérieux officiel et dominant en tout cas (la doxa lettrée et artistique dans ses innombrables variantes) qui non seulement opère la césure du corps populaire dans son « réalisme grotesque et folklorique » ${ }^{(18)}$ (y compris donc les licences textuelles autour du jeu de mots entre « pé » et «pet» et les allusions aux contes grivois voire au bestiaire obscène) ${ }^{(19)}$ :

«Toine riait de tout son cœur en se tapant sur le ventre et répondait :-Eh! la mé Poule, ma planche, tâche d'engraisser comme ça d'la volaille. Tâche pour voir. Et relevant sa manche sur son bras énorme - En v'là un aileron, la mé, en v'là un. Et les consommateurs tapaient du poing sur les tables $[\ldots] »$

Le refoulement le plus constant est celui d'une sexualité aussi imaginaire que débridée en ses fantasmes érotiques et patriarcaux ( «Il avait cette coutume d'appeler tout le monde «mon gendre »), même si notre fameux «père Toine » n' a pas d'enfant, comme on l'aura noté («Il n'eût jamais eu de fille mariée ou à marier »). Mais plus encore c'est la censure de toute allusion iconique à la religion qui anecdotise le récit et le coupe de son propre système axiologique. La profanation ${ }^{(20)}$ bouffonne est pourtant manifeste si on veut bien lire l'affrontement du pé Toine et de la mè Toine comme une transposition littéraire du combat de Carnaval contre Carême. Toine est par son nom même une figure parodique de Saint

(14) M. Bakhtine, op. cit., p. 62.

(15) Id., pp. 62-64.

(16) Id.pp. 63-64.

(17) M. Bakhtine, La poétique de Dostö̈evski, Paris, Seuil, 1970, p. 214.

(18) M. Bakhtine, L'œuvre de F. Rabelais, op. cit., p. 62.

(19) Toine est l'homme des vents (carnavalesques) : il est le héros topique du hameau de Tournevent et l'onomastique joue sans cesse de l'homophonie pé/pet. Voir J.-M. Privat, « Entre oral, anal et carnaval. Maupassant ou les ruses du dire ", à paraître.

(20) Sur la profanation et la parodia sacra comme catégorie de perception et mode d'expression de la culture de carnaval, M. Bakhtine, La poétique de Dostö̈evski, op. cit., pp. 169-175. 
Antoine, le moine ascète au désert ${ }^{(21)}$, et son aventure un double carnavalesque du saint mystère de l'enfantement virginal conjugué au miracle de Pâques (et de ses œufs) :

«Toine $[\ldots]$ demanda avec une angoisse de femme qui va devenir mère - Ce sera tantôt ? La vieille répondit [...] - Faut croire ! Ils attendirent. Les amis prévenus que les temps étaient proches [...]. Toine qui suait d'émotion, d'angoisse, d'inquiétude, murmura [...]. Et Toine commença à s'enorgueillir de cette paternité singulière [...]. C'était un drôle d'homme vraiment! Il déclara :-Ca fait six. Nom de nom què baptême. Et un grand rire s'éleva dans le public [...]. Tous les œufs étaient bons ! Et Toine, affolé de joie, délivré, glorieux [...] fut saisi par une tendresse de mère pour cet être tout petiot qu'il avait donné à la vie [...]. Les assistants, ravis, s'en allèrent en devisant de l'événement [...] et Horslaville, resté le dernier, demanda :- Tu m'invites à fricasser [...] ? A cette idée de fricasser, le visage de Toine s'illumina [...].»

Toine en homme enceint ${ }^{(22)}$ donc et en saint homme de carnaval qui se livre au rite folklorique de la couvade ${ }^{(23)}$, le motif de la « couvade » étant lui-même engendré par une argumentation farcesque :

« Il est chaud comme un four, vot'homme, qui n'sort point d'son lit. Eh ben, mé, j'li f'rais couver des œufs [...]. Pisqu'on fait ben couver d's œufs dans une boîte chaude, on peut ben en mett' couver dans un lit [...].

Toine s'inquiétait de la couveuse jaune qui accomplissait dans le poulailler la même besogne que lui. Il demandait à sa femme : - La jaune a-t-elle mangé anuit ? Et la vieille allait de ses poules à son homme et de son homme à ses poules, obsédée, possédée par la préoccupation des petits poulets qui mûrissaient dans le lit et dans le nid. »

On ne saurait donc dissocier l'histoire des illustrations d'une histoire de l'édition et de ses enjeux propres (économiques, idéologiques, institutionnels). Mais notre propos était autre, une histoire culturelle de la littérature et de la constitution iconique d'horizons d'attente qui peuvent rapidement disposer du texte, en modifier l'interprétation jusqu'à en perdre de vue les reliefs et l'horizon même ${ }^{(24)}$. Ainsi pour exemple et ce dés janvier 1886, le légitimisme lettré du chroniqueur de La Revue contemporaine ${ }^{(25)}$ :

(21) Maupassant est coutumier du fait : «On l'appelait Saint-Antoine, parce qu'il se nommait Antoine, et aussi peut-être parce qu'il était bon vivant, joyeux, farceur, puissant mangeur et fort buveur, et vigoureux trousseur de servantes, bien qu'il eût plus de soixante ans. C'était un grand paysan du pays de Caux, haut en couleur, gros de poitrine et de ventre [...]», «Saint-Antoine », Gil Blas, 3 avril 1883.

(22) Voir R. Zapperi, L'homme enceint. L'homme, la femme et le pouvoir, Paris, PUF, 1983 et J.M. Privat, «Toine. Un géant chez Maupassant», Actes du colloque « Géants, dragons et animaux fantastiques en Europe» (Ath), Tradition Wallonne, Bruxelles, 2004, pp. 65-81.

(23) Bornons-nous ici à citer la définition (très ethnocentrée) du Littré sur le sujet : « La couvade se dit encore, dans quelques parties de la France, de la coutume bizarre en vertu de laquelle, quand une femme est accouchée, le mari se met au lit, prend l'enfant, et reçoit les compliments de ses voisins. »

(24) On trouvera en Annexe quelques compléments iconographiques utiles pour la classe.

(25) Chronique de E.A, « Toine, par M. Guy de Maupassant, 1 vol. in-18, illustré par Mesplès, Marpon et Flammarion, éditeurs ", Critiques littéraires et artistiques, La Revue contemporaine, janvier 1886, pp. 119-120. 
«A quelques exceptions près, le nouveau recueil de M. Guy de Maupassant est bien fait pour désopiler les rates les plus moroses. Il y a dans l'auteur de Bel-Ami une faculté spéciale de rire et de faire rire, qui s'accorde à merveille avec l'ensemble un peu brutal de son beau talent. Sa gaieté n'est point celle de tout le monde ; elle n'est aucunement dans les mots, et réside tout entière dans l'outrance et le grotesque de situations minutieusement réelles. C'est quelque chose d'énorme et d'entraînant. Impossible de résister à son influence, à son charme [...]. Mais pourquoi faut-il que l'éditeur ait jugé à propos d'orner le volume d'illustrations insignifiantes qui augmentent le prix sans rien ajouter à sa valeur. M. Mesplès aurait bien dû se rendre compte que ses minces croquetons ne sont point d'accord avec la belle prose de M. de Maupassant. » E.A.

Les « croquetons » ont en effet quelque chose d'un peu brutal quand ils s'ajustent à la culture du texte alors que le « beau talent» de l'écrivain autoriserait toutes les euphémisations référentielles («taper sur le ventre » entrerait ainsi sagement dans l'imagerie populaire traditionnelle ou les façons de dire ordinaires, et non plus dans les façons de faire). La légitimité, c'est le style. Le style sublimerait le commun voire le trivial sinon le vulgaire populaire des situations « minutieusement réelles. » A ceci près que «l'outrance et le grotesque » sont aussi dans les « mots » de Maupassant, particulièrement dans Toine.

Et deux ans plus tard, dans le Supplément littéraire de La Lanterne ${ }^{(26)}$ le dessin (ill. 6) qui illustre en première page ${ }^{(27)}$ la scène de la couvée/couvade conserve quelque chose de la bonne grosse farce traditionnelle (et misogyne), mais l'altérité culturelle des paysans apparaît sous un jour presque plus lugubre que pittoresque, seuls les trois farceurs à l'arrière-plan jouant sur un registre plus franchement comique. Le premier tout à son étonnement réjoui porte l'habit d'un notable local, le second au bourgeron dessiné à traits sommaires semble ouvrir un bec tout populaire et le dernier, à peine esquissé et à peine visible, est croqué dans l'effarement anthropologique de l'idiot du village, un peu comme ce «Césaire Paumelle qui ne parlait jamais, mais qui s'amusait tout de même. » Comme si le dessinateur anonyme rendait à Césaire ce qui appartient à Césaire. A lui seul, déjà. Comme si le bon bougre était relégué à l'arrière-plan du monde « toinesque », dans une image qui condense ce qu'il allait advenir dans l'histoire éditoriale des lectures illustrées de Toine.

(26) Le Supplément littéraire, 5 août 1888. Le texte de Toine avait été repris dans La Vie populaire (7 mars 1886), dans Histoire d'une fille de ferme (choix de contes constituant le numéro 111 des «Auteurs célèbres », Flammarion, 1890) et dans le Supplément du Petit Journal (19 mars 1892).

(27) La Lanterne. Lancé en avril 1877 par le financier Eugène Mayer, ce quotidien est la seule réussite du journalisme radical. Journal populaire, il connaît un franc succès, tirant dès ses débuts à plus de 100.000 exemplaires. Il doit essentiellement sa célébrité à son anticléricalisme virulent, et aux campagnes que ses rédacteurs mènent contre la police parisienne qui lui valent de multiples condamnations, et dont résulte la démission du préfet de police et du ministre de 1'Intérieur. On compte parmi ses directeurs et rédacteurs Briand, Millerand, Viviani, ou encore Jaurès et Pelletan. La Lanterne voit son succès décroître après la guerre, sa publication se poursuivant toutefois jusqu'en 1938. 


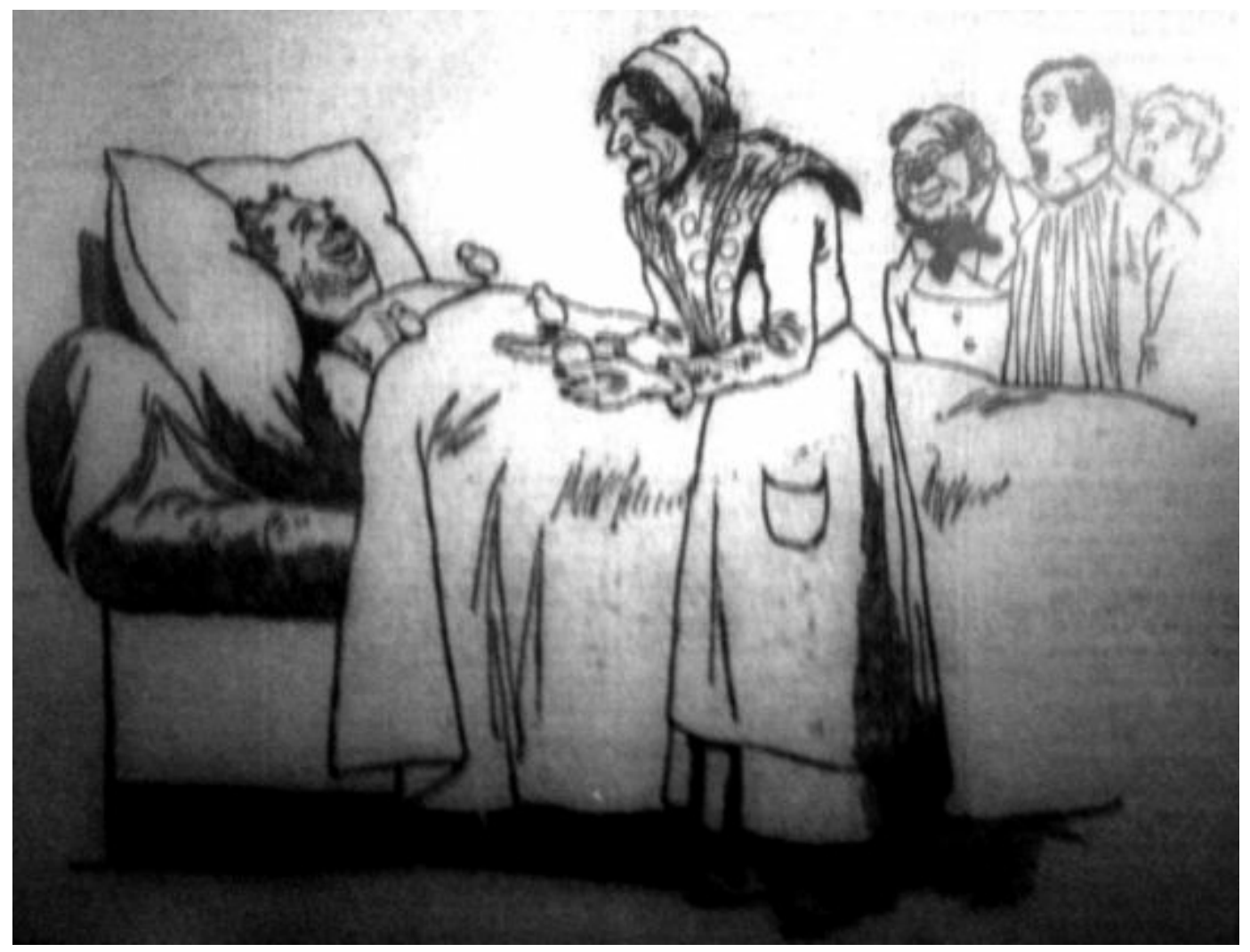

Illustration 6 : 1888. La Lanterne. Supplément littéraire, 5 août 1888, avec un dessin anonyme. Cliché personnel reproduit d'après microfilm ; avec l'aimable autorisation de la Bibliothèque Sainte-Geneviève, Paris. 


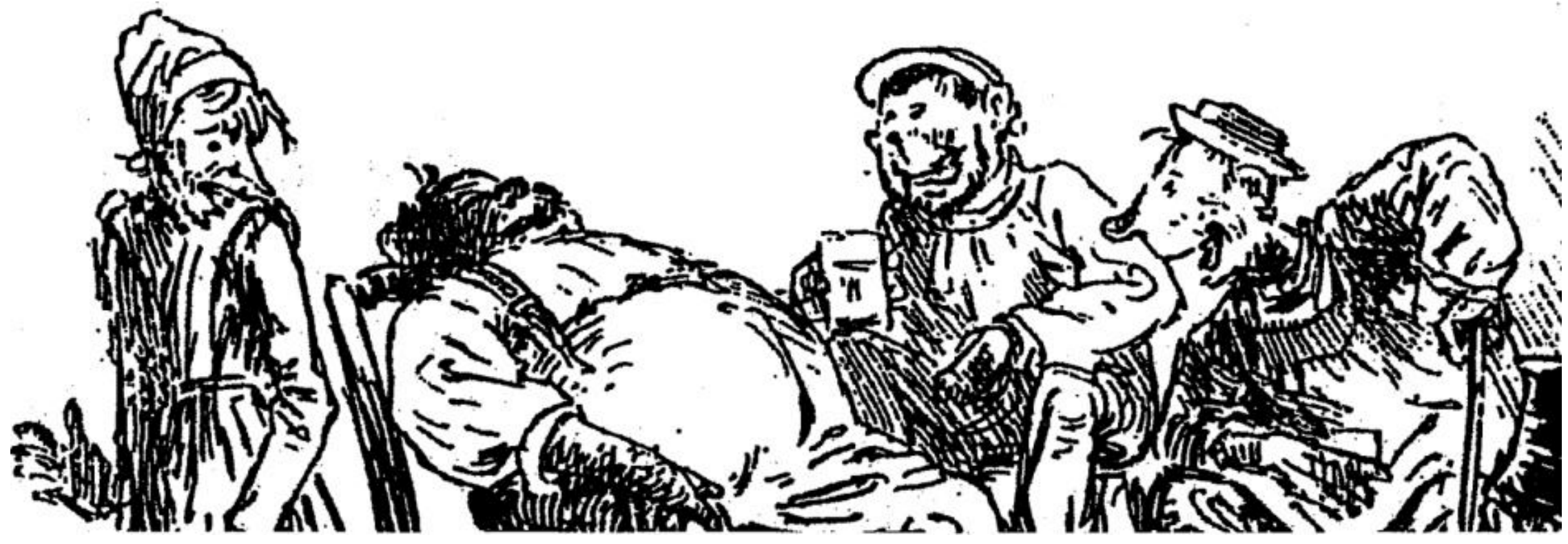

Illustration 1 : 1886. Toine, Edition originale, Illustration P.-E. Mesplès, Paris, Bibliothèque illustrée à 5 francs le volume, Marpon \& Flammarion, 1886. (Détail agrandi par nos soins) 


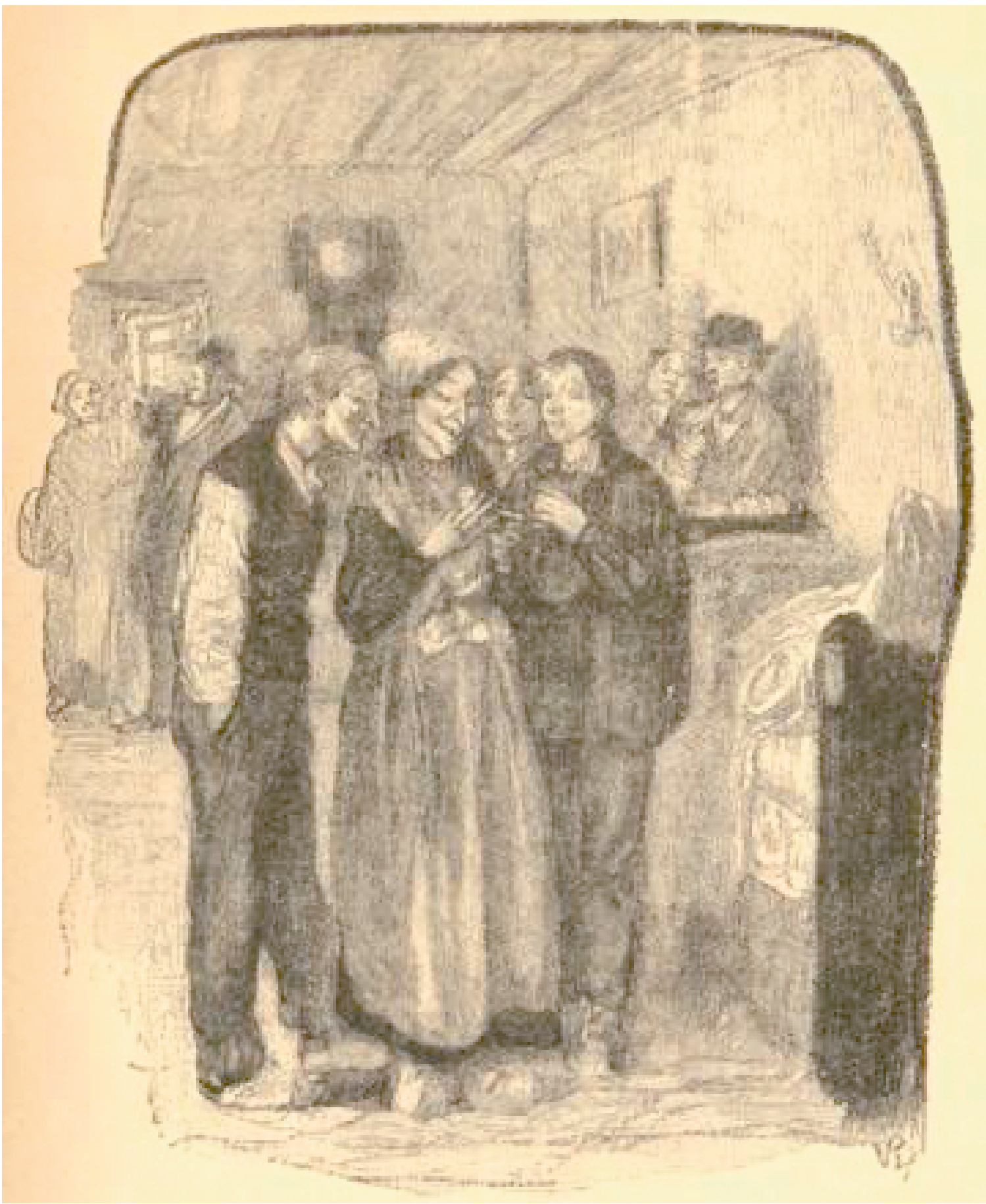

Illustration 2 :1903. Toine, Illustration V. Rottembourg, gravée sur bois par G. Lemoine, Paris, Ollendorff, Euvres complètes illustrées de Guy de Maupassant, 1903. 


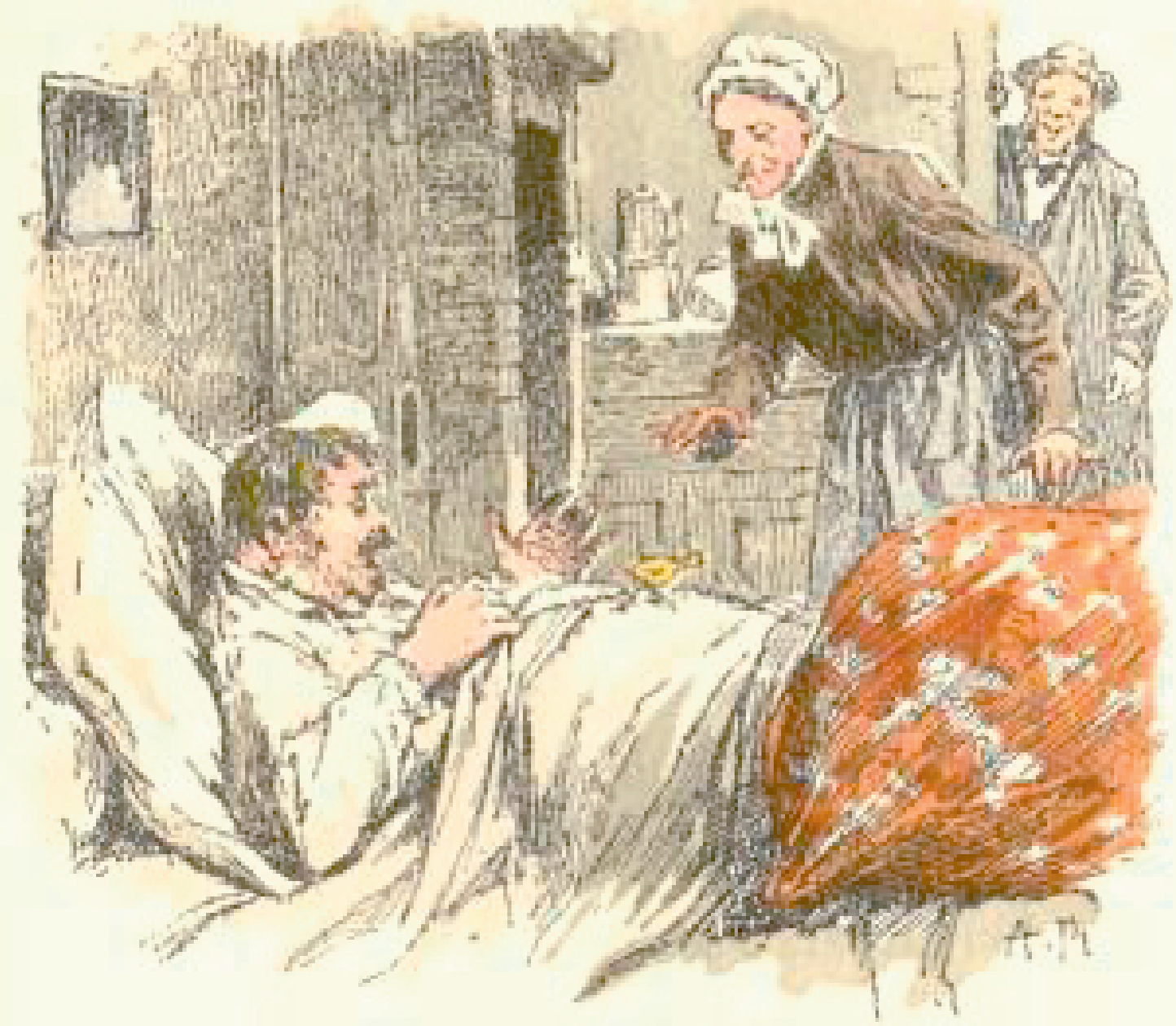

Illustration 3 :1923. Toine suivi de Histoire d'une fille de ferme, Illustrations en couleurs par A. Robaudi, Paris, A. Ferroud \& F. Ferroud, 1923 


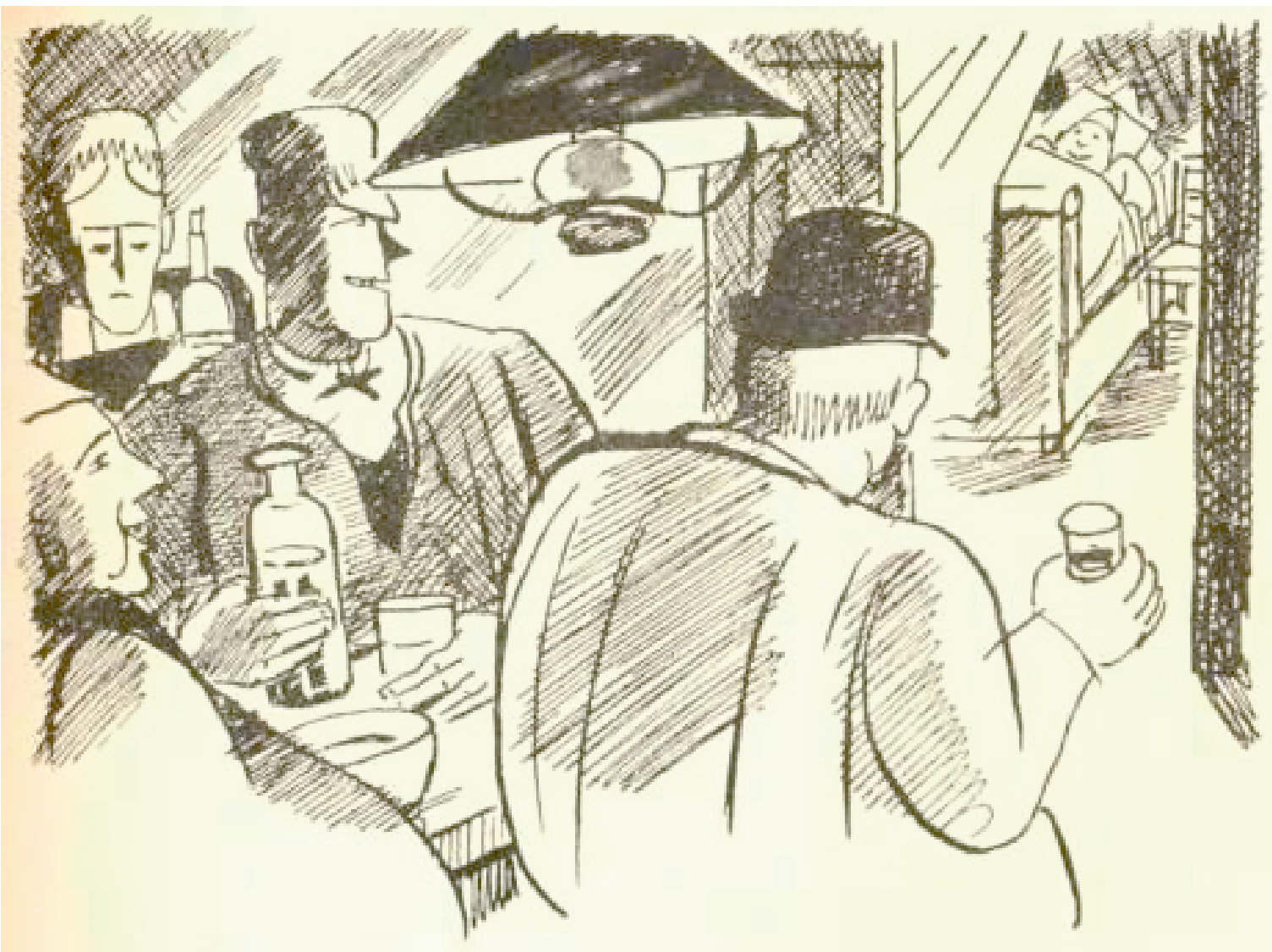

Illustration 4 : 1938. Toine, Illustrations P. Falké, Paris, Librairie de France, CEuvres complètes illustrées de Guy de Maupassant, V, 1938. 


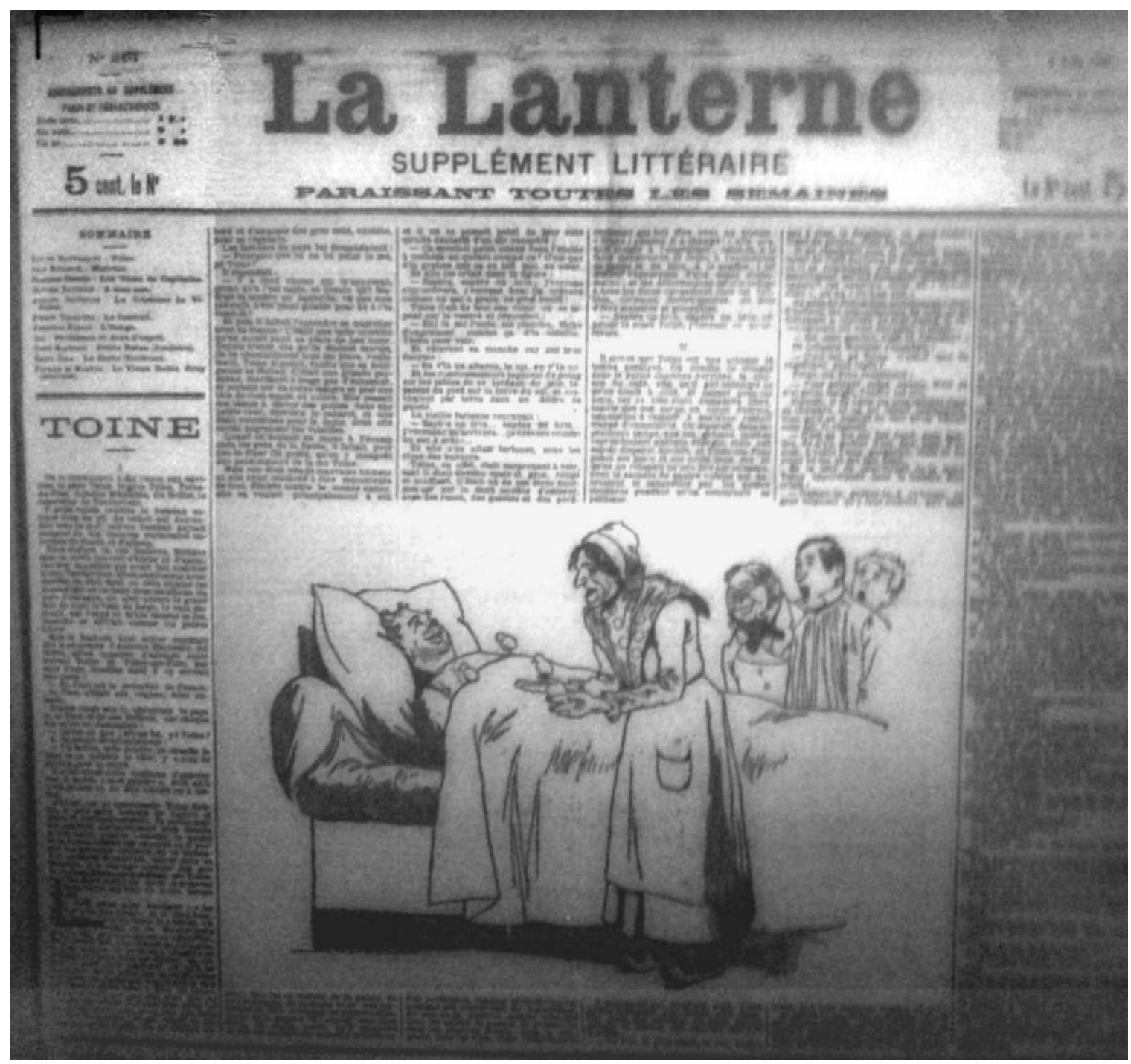

Illustration 5 : 1888. La Lanterne. Supplément littéraire, 5 août 1888, avec un dessin anonyme. Cliché personnel reproduit d'après microfilm ; avec l'aimable autorisation de la Bibliothèque SainteGeneviève, Paris. 